\title{
Patient's perspective of sustained remission in rheumatoid arthritis
}

\author{
Irazú Contreras-Yáñez ${ }^{1}$, Guillermo Guaracha-Basañez ${ }^{2}$, Daniel Ruiz-Domínguez ${ }^{1}$ and Virginia Pascual-Ramos ${ }^{1 *}$ (D)
}

\begin{abstract}
Background: During the course of rheumatoid arthritis (RA), patients have profound negative effects on their patient-reported-outcomes (PRO); in addition, the impact of sustained remission (SR) on PROs may differ for each particular outcome. The objectives of this study were to identify SR from an inception cohort of RA patients and to examine the impact of SR in an ample spectrum of PROs.

Methods: The study was developed in a well characterized and ongoing cohort of RA patients with recent onset disease recruited from 2004 onwards. In November 2016, the cohort included 187 patients, of whom 145 had at least 30 months of follow-up, with complete rheumatic assessments at regular intervals in addition to a pain visual analogue scale (PVAS), overall disease-VAS (OVAS), health assessment questionnaire (HAQ), Short-Form 36v2 Survey (SF-36) and fatigue assessment. First SR was defined according to the DAS28 cut-offs (DAS28-SR) and ACR/EULAR 2011 Boolean definition (B-SR), if maintained for at least 12 consecutive months. The dependent $t$ test and Mc Nemar's tests were used for comparisons between related groups. Local IRB approval was obtained.

Results: More patients achieved DAS28-SR than B-SR: 78 vs. 63, respectively. Fifty patients met both SR definitions. Follow-up to DAS28-SR was shorter than to B-SR and the duration of DAS28-SR was longer, $p \leq 0.023$ for all comparisons.

At SR, patients had PRO proxy to normal values; the percentage of patients with normal PRO varied from $97 \%$ (95\% Cl: 91-99) for HAQ to 50\% (95\% Cl: 39-61) for absence of fatigue.

In DAS28-SR patients, acute reactant phases within the normal range were detected very early (after 1.5-2.

9 months). HAQ, PVAS, OVAS and SF-36 were scored within the normal range after 6-7 months. The absence of fatigue was detected at 8.7 months of follow-up, which was similar to DAS28-SR. In the 63 patients with B-SR, a similar pattern was observed. The follow-up to outcomes of the 50 patients who met both SR definitions was similar, but the absence of fatigue and physical component SF-36 normalization were achieved earlier in B-SR patients $(p \leq 0.02)$.
\end{abstract}

Conclusions: The impact of SR on PRO differs accordingly to each particular outcome.

Keywords: Patient-reported-outcomes, Remission, Rheumatoid arthritis

\section{Background}

Clinical remission has become a widely accepted treatment goal in rheumatoid arthritis (RA) patients with early disease. Reaching remission, however, is only the first step in a targeted treatment approach, and sustained remission (SR) is the desirable subsequent step. Previous studies have demonstrated that the progression

\footnotetext{
* Correspondence: virtichu@mail.com

'Department of Immunology and Rheumatology, Instituto Nacional de Ciencias Médicas y Nutrición Salvador Zubirán, Vasco de Quiroga 15, Colonia Belisario Domínguez, Sección XVI, 14080 México City, CP, Mexico

Full list of author information is available at the end of the article
}

of joint damage decreases with increasing duration of remission [1]; nonetheless, the ultimate target to drive treatment decisions in RA patients should be better long-term patient-reported outcomes [2].

In the last few decades, international efforts had been committed to defining remission in RA. Various definitions are available, with different levels of stringency [3-5]. Ultimately, in RA patients, remission may be operationalized as either a complete absence of disease activity or a level of disease activity that is so low that it is not troublesome to the patient and portends a good prognosis [6]. 
This definition is particularly relevant given that during the course of RA, affected individuals face considerable physical, psychological and social changes that have profound negative effects on their health-related QoL (HRQoL) [7]. However, when remission is achieved, RA patients also achieved population norms in terms of HRQoL, unlike in the case of patients with other rheumatic diseases, such as patients with ANCA-associated vasculitides in remission, who exhibit substantially reduced patient-reported outcomes (PRO) [8].

Current RA management guidelines recommend incorporating patient-reported measures of functioning and quality of life into clinical trials as they are as effective as traditional physician- or laboratory-reported outcomes in reflecting long-term morbidity and mortality, easier to administer and less expensive than physician-observed health status measures [9-11]. Moreover, clinicians and patients have different perspectives, and patients prioritize clinical outcomes that are not routinely measured $[10,12]$. PROs include outcomes that are limited to a symptom (such as pain or fatigue) or those that evaluate complex constructs, such as patient's function, disability or the overall disease status. One relevant aspect of the impact of SR on PROs is that it may differ for each particular outcome. In the present study, we sought to identify SR patients from an inception and ongoing cohort of RA patients with recent onset disease at cohort enrollment and examine the impact of SR in an ample spectrum of PROs.

The specific objectives were as follows:

1. - To describe PROs from RA patients who achieved SR for the first time as well as the proportion of those patients who achieved PRO norms according to 2 definitions of SR.

2. - To describe the time to normalization of PROs in RA patients who achieved SR.

\section{Methods}

\section{The early RA cohort}

In February 2004, an ongoing cohort of patients with recent-onset RA (within 12 months of symptoms onset) was initiated. At inclusion, the complete medical history and sociodemographic data were recorded in addition to the type(s) and levels of rheumatoid factor (RF) and antibodies against cyclic citrullinated proteins (ACCP). Consecutive medical evaluations were standardized and scheduled at regular intervals: 2 months apart for the first 2 years of follow-up and then 2, 4 or 6 months apart depending on patient and disease characteristics. When patients required additional consultations because adverse events or unexpected conditions (pregnancy, flares, comorbidity, etc....) a visit with the attending rheumatologist was scheduled within 1 week.
Clinical assessments always included at least swollen and tender joint counts, physician overall disease activity, erythrocyte sedimentation rate (ESR) and C-reactive protein (CRP) level, comorbidities (established by a record review) and Charlson score [13], and treatment assessments. In addition, the following PROs were evaluated before clinical assessments that were performed by a single rheumatologist: a pain visual analogue scale (P-VAS), overall disease-VAS (O-VAS), health assessment questionnaire (HAQ) [14], Short-Form 36v2 Survey (SF-36) $[15,16]$ and (presence/absence) of fatigue.

Patients had health expenditures government coverage depending on their incomes but needed to pay for their medication and these were not provided by the local pharmacy (unless patients were hospitalized and if available). Treatment was prescribed by the rheumatologist in charge of the clinic, was "Treat-to-target" oriented and according to the current standard of care (no protocol was followed). Traditional Disease Modifying AntiRheumatic Drugs (DMARDs) were used in $98 \%$ of them with/without corticosteroids (50\% of patients received low doses of oral corticosteroids during their follow-up). Subcutaneous methotrexate was the first DMARD indicated (unless contraindicated) although most of the patients (72\%) had 2-3 combined DMARDs during their follow-up [17]. When flares were identified, corticosteroids and/or DMARDs were added or doses increased (if suboptimal). Patient's preferences and resources were always considered.

In November 2016, the cohort comprised 187 RA patients with a variable follow-up recruited from 2004 onwards. Of these patients, 145 had at least 30 months of follow-up (baseline and consecutive visits numbered as 2 to 16), which was deemed to be convenient to accomplish the objectives described, including achieving a SR state. We previously reported that patients from the Early Arthritis Clinic (EAC) achieved their first sustained remission state at $14 \pm 9$ months of follow-up [18].

\section{Definitions}

Sustained remission (SR) was defined according to disease activity score on 28 joints (DAS28) cut-offs (DAS28-SR) and according to the American College of Rheumatology (ACR)/ European League Against Rheumatism (EULAR) 2011 Boolean definition (B-SR).

First DAS28-SR was considered (Yes/No) to occur when patients achieved at least 12 months of continuous follow-up with DAS28 $<2.4$ for the first time [3, 19].

First B-SR was considered (Yes/No) to occur when patients achieved at least 12 months of continuous follow-up including swollen a 28 -joint count $\leq 1$ AND 
tender 28-joint count $\leq 1$ AND CRP $\leq 1 \mathrm{mg} / \mathrm{dL}$ AND patient O-VAS $\leq 1(0-10 \mathrm{~cm})$ for the first time [4].

Time in $S R$ was computed from the first visit (time) that SR was achieved up to the last follow-up, with SR according to the definition used.

HAQ norm (HAQ-N) was considered if $\leq 0.25$ on a scale from 0 to 3 [20].

SF-36 norm (SF-36-N, global score and either mental or physical scores) was considered if $\geq 80$ on a scale from 0 to 100 . The cut-off was derived from data obtained from a healthy Mexican population [21].

PVAS and OVAS norms (PVAS-N and OVAS-N) were defined as $\leq 10$ on a scale from 0 to $100 \mathrm{~mm}$ [22].

Substantial fatigue (presence/absence) was defined after re-scoring the vitality domain of the SF-36v2 domain. Data are presented as a continuous variable (on a 0 to 100 scale) and a dichotomus variable (absence/ presence). Substantial fatigue was arbitrarily defined if the mean of the four items included in the vitality domain was $<80$.

The cut-offs for ESR (Westergren method) were $<30 \mathrm{~mm} / \mathrm{H}$ for females or $<20 \mathrm{~mm} / \mathrm{H}$ for males [5].

The cut-off for CRP (Nephelometry) was defined as $\leq 1.57 \mathrm{mg} / \mathrm{dL}$ according to manufacturer's recommendations (Beckman Coulter, Inc.).

Physician overall disease VAS normalization (PhyVAS-N) was defined as $\leq 10 \mathrm{~mm}$ on a scale from 0 to 100 .

PROs during SR were recorded at the same time point: at least 6-months of follow-up with SR before and after PROs were recorded.

\section{Statistics}

Descriptive statistics was used, number and percentage (with 95\% CI) for dichotomus variables and (mean \pm SD) for continuous variables. The dependent $t$ test and $\mathrm{Mc}$ Nemar's tests were used for comparisons between related groups, either as percentages or the (mean $\pm \mathrm{SD}$ ). Gender has been associated to unfavorable outcomes [23] and analysis were repeated in female and male subpopulations. Relevant results are presented.

All statistical tests were 2-sided and evaluated at the 0.05 significance level. Statistical analysis was performed using the SPSS/PC program (v.17.0; Chicago IL).

\section{Ethics}

The present study was approved by the Institution's internal review board "Comité de ética del Instituto Nacional de Ciencias Médicas y Nutrición Salvador Zubirán", with the reference number IRE-274-10/11-1. Written informed consent was obtained from all patients to have their charts reviewed and data presented in scientific forums or published.

\section{Results}

Characteristics of the entire population and comparison of females and males patients with DAS28-SR and with B-SR.

The entire population comprised 145 RA patients, among whom 130 (89.7\% [85-95]) were females; their data are summarized in Table 1 (first column).

In the entire population, an increased number of patients achieved DAS28-SR compared to B-SR, 78 vs. 63 patients out of 145 , respectively. There were 50 patients who met bot definitions of SR. In those patients, followup to DAS28-SR was shorter than to B-SR and the duration of DAS28-SR was longer than the duration of B-SR. Similar results were obtained in the subpopulation of females and a similar tendency was seeing in the subpopulation of males but differences did not showed statistical significance due to the limited number of male patients (data not shown). Table 2 summarizes results.

Table 1 also compares baseline and cumulative characteristics between females and males patients with DAS28-SR and B-SR. At cohort inclusion, no differences were found but B-SR males were 10 years older than their female counterpart ( $45.6 \pm 6.3$ vs. $35.6 \pm 12.2$ years, $p=0.01$ ); cumulative (up to SR) treatment and comorbidity were also similar but DAS28-SR males had higher number of comorbidities/patients than their female counterpart ( $1.5 \pm 0.8$ vs. $1.3 \pm 0.8, p=0.03)$. Data are summarized in Table 1 .

\section{PRO in DAS-28 SR and B-SR patients}

The following (mean \pm SD) PRO at either DAS28-SR or B-SR are summarized in Table 3: PVAS, OVAS, tender joints count, HAQ, physical and mental component of the SF-36 and fatigue. In general, at SR, patients had PRO proxy to normal values. In addition, the percentage $(95 \% \mathrm{CI})$ of patients with normal PRO varied from $97 \%$ (91-99) for HAQ-N, but decreased to 50\% (39-61) for absence of fatigue, as shown in Fig. 1. There were no differences between females and males PRO scores at either DAS28-SR or B-SR (data not shown).

We compared the number (\%) of patients who achieved PRO norms within the 50 patients who met DAS28-SR and B-SR, and no differences were noted. However, B-SR patients achieved a higher SF-36 mental component compared with their counterparts $(92.3 \pm 7.4$ vs. $90.6 \pm 0.1, p=0.041)$.

\section{Months of follow-up to achieve outcome norms in patients with DAS28-SR and B-SR}

In the population of DAS28-SR patients, we calculated the (mean $\pm \mathrm{SD}$ ) months of follow-up to achieve the norm for each PRO; we also determined the time to achieve values within the normal range of ESR and CRP and the time to achieve $\leq 1$ swollen joint and PhyVAS-N. The results from the entire population are summarized 
Table 1 Baseline and cumulative characteristics from the entire population and comparison of females and males patients with DAS28-SR and with B-SR

\begin{tabular}{|c|c|c|c|c|c|c|}
\hline & \multirow{2}{*}{$\begin{array}{l}\text { Entire population } \\
N=145\end{array}$} & \multicolumn{2}{|l|}{ Females, $N=130$} & \multicolumn{2}{|l|}{ Males, $N=15$} & \multirow[t]{2}{*}{$p^{1} / p^{2}$} \\
\hline & & $\begin{array}{l}\text { DAS28-SR } \\
N=68\end{array}$ & $\begin{array}{l}\text { B-SR } \\
N=55\end{array}$ & $\begin{array}{l}\text { DAS28-SR } \\
N=10\end{array}$ & $\begin{array}{l}\text { B-SR } \\
N=8\end{array}$ & \\
\hline \multicolumn{7}{|l|}{ Baseline characteristics $^{a}$} \\
\hline Age, years & $37.9 \pm 12.9$ & $34.3 \pm 11.5$ & $35.6 \pm 12.2$ & $36.9 \pm 12$ & $45.6 \pm 6.3$ & $0.22 / 0.01$ \\
\hline Years of formal education & $11.1 \pm 3.9$ & $12 \pm 3.8$ & $11.9 \pm 3.7$ & $11.8 \pm 3.6$ & $10.6 \pm 2.6$ & $0.39 / 0.29$ \\
\hline $\mathrm{N}^{\circ}(\%[95 \% \mathrm{CI})$ of patients RF+ & $118(81.4[75-88])$ & $52(76.5[66-87])$ & $43(78.2[67-89])$ & $9(90$ [30-95]) & 8 (100 [N.A.]) & $0.45 / 0.33$ \\
\hline $\mathrm{N}^{\circ}(\%[95 \% \mathrm{CI}])$ of patients ACCP+ & 123 (84.8 [79-91]) & $54(79.4[70-89])$ & $43(78.2[67-89])$ & $9(90[30-65])$ & $7(87.5[25-89])$ & $0.68 / 1$ \\
\hline Symptoms duration, months & $5.4 \pm 2.6$ & $5.5 \pm 2.7$ & $5.4 \pm 2.3$ & $5.3 \pm 2.2$ & $4.3 \pm 1.4$ & $0.39 / 0.16$ \\
\hline DAS28 & $5.9 \pm 1.4$ & $5.6 \pm 1.5$ & $5.6 \pm 1.5$ & $5.6 \pm 1.4$ & $5.5 \pm 0.7$ & $0.59 / 0.58$ \\
\hline $\begin{array}{l}\mathrm{N}^{\circ}(\%[95 \% \mathrm{CI}]) \text { of patients with } \\
\text { DMARDs at referral }\end{array}$ & $37(25.5$ [18-33]) & $17(25[15-35])$ & $15(27.3[16-39])$ & $4(40[10-70])$ & $2(25[5-55])$ & $0.27 / 1$ \\
\hline $\mathrm{N}^{\circ}(\%[95 \% \mathrm{Cl}])$ of patients with CTs at referral & $40(27.6[20-35])$ & $22(32.4[21-44])$ & $13(23.6[12-35])$ & $4(40[10-70])$ & $2(25[5-55])$ & $0.72 / 1$ \\
\hline $\mathrm{N}^{\circ}(\%[95 \% \mathrm{Cl}])$ of patients with comorbidity & $60(41.4[33-95])$ & $33(48.5[37-60])$ & $28(50.9[38-64])$ & $7(70[10-71])$ & $5(62.5[5-65])$ & $0.31 / 0.71$ \\
\hline Charlson score & $1.4 \pm 0.6$ & $1.5 \pm 0.6$ & $1.5 \pm 0.6$ & $1.6 \pm 0.7$ & $1.8 \pm 0.7$ & $0.08 / 0.26$ \\
\hline \multicolumn{7}{|l|}{ Cumulative (up to SR) characteristics } \\
\hline DAS28 & Not aplicable & $2.3 \pm 0.6$ & $2.3 \pm 0.6$ & $2.3 \pm 0.7$ & $2.5 \pm 0.9$ & $0.99 / 0.59$ \\
\hline$N^{\circ}$ DMARD/patient & $1.8 \pm 0.7$ & $1.7 \pm 0.5$ & $1.7 \pm 0.5$ & $1.6 \pm 0.6$ & $1.5 \pm 0.5$ & $0.4 / 0.29$ \\
\hline $\mathrm{N}^{\circ}(\%[95 \% \mathrm{CI}])$ of patients with CTs & $58(40[32-48])$ & $28(41.2[30-53])$ & $21(38.2[25-51])$ & $5(50[19-81])$ & $2(25[5-55])$ & $0.76 / 1$ \\
\hline $\mathrm{N}^{\circ}$ of comorbidity/patient & $1.6 \pm 0.8$ & $1.3 \pm 0.8$ & $1.5 \pm 0.9$ & $1.5 \pm 0.8$ & $1.8 \pm 0.7$ & $0.03 / 0.26$ \\
\hline
\end{tabular}

$\mathrm{p}^{1}=$ Comparison of DAS28-SR females and DAS28-SR males

$\mathrm{p}^{2}=$ Comparison of B-SR females and B-SR males

$\mathrm{Cl}=$ Confidence interval. $\mathrm{RF}=$ Rheumatoid factor. $\mathrm{ACCP}=$ Antibodies to cyclic citrullinated peptides. DAS28 = Disease activity index (28 joints evaluated).

DMARDs = Disease modifying anti-rheumatic drugs. CTs $=$ Corticosteroids

${ }^{\mathrm{a}}$ Data presented as (mean $\pm \mathrm{SD}$ ) and number (percentage $[95 \% \mathrm{Cl}]$ )

in Table 4. The number of patients with DAS28-SR who achieved the norm varied for each outcome, from 34 patients who referred absence of substantial fatigue to 77 patients with PVAS-N, PhyVAS-N and ESR-N. Interestingly, normalization of ESR and CRP was detected very early (at 1.5 and 2.9 months from baseline, respectively), followed by HAQ-N, PVAS-N, OVAS-N, SF-36-N (achieved between 6 and 7 months of followup), and tender joint count $\leq 1$ (at 7.6 months). Absence of fatigue was detected late, at 8.7 months of follow-up, similar to DAS28-SR, which was achieved at (mean) 9.8 months (Fig. 2).

In the 63 patients with B-SR, a similar pattern was observed in the number of patients who achieved each particular outcome. In addition, normalization of the acute reactant phases was identified early during followup, followed by HAQ-N, PVAS-N, OVAS-N and both components of the SF-36. Of note, absence of fatigue was detected at 6.4 months of follow-up, before swollen and tender joint counts and PhyVAS-N (Table 4, Fig. 2).

Finally, we compared the follow-up to outcomes within the 50 patients who met both SR definitions. No differences were observed between both groups, but absence of substantial fatigue and physical component SF-36-N were achieved significantly earlier in B-SR patients $(p \leq 0.02)$.

\section{Discussion}

Remission (sustained) has become the best clinical outcome in RA patients and may be approached as a state

Table 2 Comparison of DAS28-SR and B-SR in the entire population

\begin{tabular}{llll}
\hline & DAS28-SR $^{\text {a }}$ & B-SR $^{\text {a }}$ & $p$ \\
\hline$N^{\circ}(\%[95 \%$ Cl]) of patients who achieved SR & $78(53.8[46-62])$ & $63(43.4[35-52])$ & 0.000 \\
Months of follow-up to SR $^{b}$ & $9.1 \pm 5$ & $10.9 \pm 5.6$ & 0.023 \\
Months of SR duration $^{b}$ & $22.3 \pm 5.7$ & $17.5 \pm 6.2$ & 0.000 \\
\hline
\end{tabular}

$\mathrm{Cl}=$ Confidence interval. DAS28-SR = Sustained remission according to the Disease activity index (28 joints considered). B-SR $=$ Boolean sustained remission. $\mathrm{SR}=$ Sustained remission

${ }^{\mathrm{a}}$ Data presented as (mean $\pm \mathrm{SD}$ ) and number (percentage $[95 \% \mathrm{CI}]$ )

${ }^{\mathrm{b}}$ Data restricted to 50 patients who met bot definitions of SR. 
Table 3 (Mean \pm SD) scores at DAS28-SR and B-SR

\begin{tabular}{lll}
\hline & DAS28-SR & B-SR \\
& $N=78$ & $N=63$ \\
\hline PVAS (0-100 mm) & $2.5 \pm 3.3$ & $1.7 \pm 2$ \\
OVAS (0-100 mm) & $2.2 \pm 3$ & $1.6 \pm 2.1$ \\
Tender joint count (0-68 joints) & $0.2 \pm 0.6$ & $0.06 \pm 0.3$ \\
HAQ (0-3) & $0.04 \pm 0.2$ & $0.12 \pm 0.8$ \\
Mental component of the SF-36 (0-100) & $89.3 \pm 11.5$ & $91.1 \pm 8.5$ \\
Physical component of the SF-36 (0-100) & $86.9 \pm 9.6$ & $87.9 \pm 8.9$ \\
Fatigue (0-100) & $76.9 \pm 15.6$ & $76.4 \pm 17.6$
\end{tabular}

DAS28-SR = Sustained remission according to the Disease activity index (28 joints considered). B-SR = Boolean sustained remission. PVAS $=$ Pain visual analogue scale. OVAS $=$ Overall disease visual analogue scale. $\mathrm{HAQ}=$ Health assessment questionnaire. SF = Short-Form 36v2 Survey

${ }^{a}$ Data presented as (mean \pm SD)

of no or minimal inflammatory disease activity associated with a halt of progression of joint damage [1], better PROs, working productivity and lower costs [24]. Today, it is a feasible target and the first recommendation from the "Treat-to-Target" approach [25]; nonetheless, patient's perception of SR does not necessarily correspond to the physician construct of SR.

In the present study, we examined the impact of SR on PROs in a real clinical setting of an ongoing early arthritis clinic where completed, periodic and standardized assessments were performed by a dedicated rheumatologist. Two definitions of SR were used with a different degree of strictness [26] that allowed us to compare how each construct affected patient's health related quality of life. The PRO examination included those adopted through consensus by the ACR, EULAR and Outcomes measures in Rheumatology (OMERACT) as well as fatigue, which was more recently added based on input from patient research partners [27, 28]. In addition, acute reactant-phase determinations and physician-derived measures were also included for additional (instead of a competitive) interpretation of the data.

We first found that SR was achieved in a substantial proportion of patients (i.e., $43 \%$ and $54 \%$, depending on the definition used), and up to $35 \%$ met both definitions. In early disease, up to $60 \%$ of patients achieve clinical remission according to DAS28 [2, 29, 30]. We found a high percentage of B-SR and performed a 28-joint count, although it is recommended to include feet and ankle assessments when applying these criteria [4]. An extended joint count may favor the eventual detection of additional joints as either tender or swollen. Similar to previous publications, the highest percentage of SR patients was achieved with the DAS28 cut-off [8, 26, 29]. We additionally found that follow-up to first SR and time in SR favor DAS28-SR that was easier to achieve and maintain. DAS28 is considered to have lesser stringency than the Boolean definition of remission [29-31]. The more stringent DAS28 cutoff value (2.4 instead of 2.6), allowed the presence of up to 12 swollen joints while the

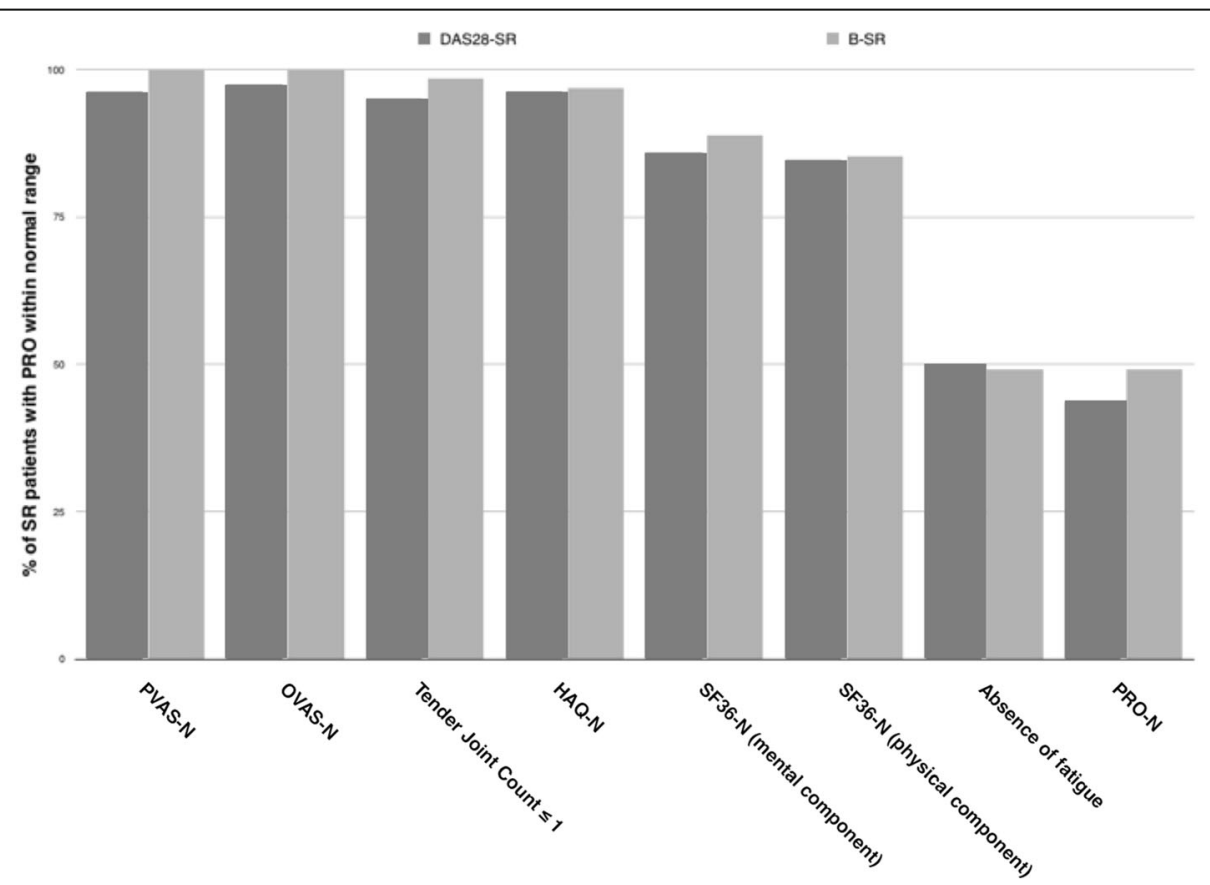

Fig. $1 \mathrm{~N}^{\circ}(\%)$ of patients who achieved PRO norms. Figures depicts the number (bottom of the bars) and percentage of DAS28-SR patients (dark grey bar) and B-SR patients (light grey bar) who achieved PVAS, OVAS, HAQ and SF-36 norms, $\leq 1$ tender joints, absence of fatigue and all PRO norms (PRO-N) 
Table 4 (Mean \pm SD) months of follow-up to outcome normalization in the 78 patients with DAS28-SR and the 63 patients with B-SR

\begin{tabular}{|c|c|c|}
\hline $\begin{array}{l}\text { Outcome, ( } \mathrm{N}^{\circ} \text { of patients who } \\
\text { achieved norm in DAS28-SR/B-SR patients) }\end{array}$ & $\begin{array}{l}\text { Months of follow-up to outcome } \\
\text { normalization in } 78 \text { DAS28-SR patients }^{\text {a }}\end{array}$ & $\begin{array}{l}\text { Months of follow-up to outcome } \\
\text { normalization in } 63 \text { B-SR patients }\end{array}$ \\
\hline ESR-N, (77/61) & $1.5 \pm 3$ & $2 \pm 4$ \\
\hline CRP-N, (73/63) & $2.9 \pm 4.4$ & $2.4 \pm 3.7$ \\
\hline HAQ-N, (75/63) & $6 \pm 5.1$ & $4.8 \pm 4.2$ \\
\hline PVAS-N, (77/63) & $6.1 \pm 4.6$ & $4.8 \pm 3.2$ \\
\hline OVAS-N, (76/63) & $6.1 \pm 4.7$ & $4.9 \pm 3.4$ \\
\hline SF-36-N, mental component, (67/54) & $6.2 \pm 4.7$ & $5.6 \pm 4.3$ \\
\hline SF-36-N, physical component, (60/48) & $7 \pm 5.5$ & $5 \pm 3.3$ \\
\hline Tender joint count $\leq 1$ (0-68 joints), (76/63) & $7.6 \pm 4.9$ & $7 \pm 4.5$ \\
\hline Swollen joint count $\leq 1$ (0-66 joints), (72/63) & $7.7 \pm 4.8$ & $7.7 \pm 4.9$ \\
\hline Physician overall disease $\leq 10(0-100 \mathrm{~mm}),(77 / 63)$ & $8.6 \pm 4.4$ & $8.1 \pm 4.6$ \\
\hline Absence of substantial fatigue, (34/24) & $8.7 \pm 5.4$ & $6.4 \pm 4.3$ \\
\hline
\end{tabular}

$\mathrm{N}^{\circ}=$ Number. DAS28-SR $=$ Sustained remission according to the Disease activity index (28 joints considered). B-SR = Boolean sustained remission. ESR-N = Erythrocyte sedimentation rate within norms. CRP- $\mathrm{N}=$ C-reactive protein within norms. HAQ-N = Health assessment questionnaire within norms. P-VAS-N = Pain visual analogue scale within norms. O-VAS-N = Overall disease-visual analogue scale within norms. SF-36- $\mathrm{N}=$ Short-Form 36v2 survey within norms

${ }^{\mathrm{a}}$ Data presented as (mean $\pm \mathrm{SD}$ )

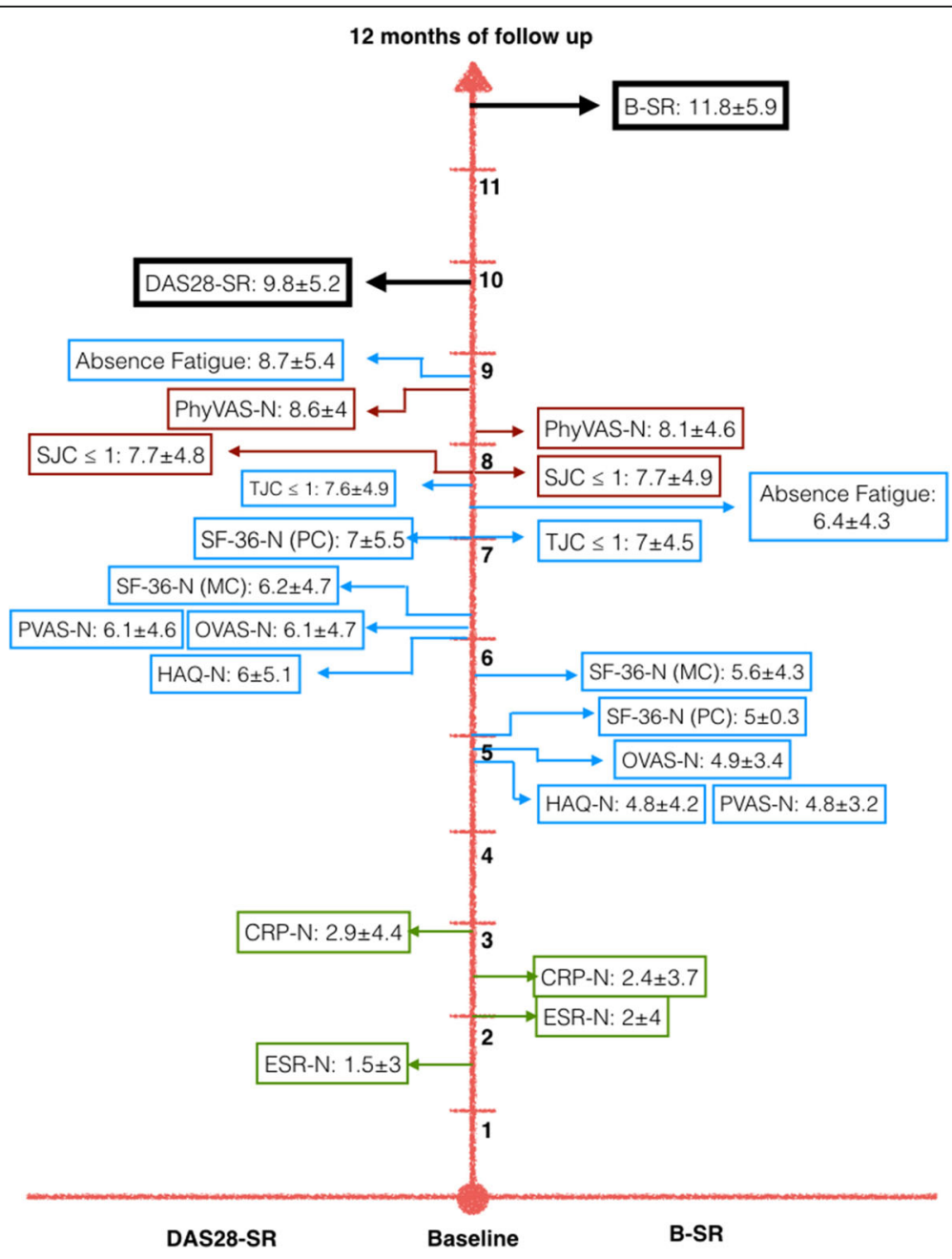

Fig. 2 Months of follow-up to achieve outcomes norms in patients with DAS28-SR and B-SR 
ESR was restricted to practically normal values; in addition, it is possible to have up to 5 swollen joints and still being in remission using the DAS28 criteria if the other variables are nearly normal [29]. Finally, DAS28 was also less stringent with respect to elevation of the global assessments of activity and pain as compared to an other validated index, the Simplified Disease Activity Index (SDAI) [32].

Second, at SR, patients from our study had PRO proxy to normal values. Linde et al. [8] found that the healthrelated quality of life scores from Danish RA patients in clinical remission approached that of the general population. By contrast, Radner et al. [24] showed that patients in remission, on average, did not reach normal healthrelated quality of life values (from HAQ, SF-36 physical component, EURO Qol-5D and Short Form-6D). Important considerations to be highlighted included that their patients had almost 12 years of disease duration with the consequent accrual of irreversible damage and disability [33] and their population was almost 25 years older than ours. Physical function declines with age [34], and increased age reduces HRQoL in aged RA patients [35]. Finally, in our study, SR (instead of time point remission) was defined, and it has been shown that physical function (as per HAQ) continues to improve over time if remission is reached and sustained [36].

Third, the percentage of patients with normal PRO varied from $97 \%$ for the $\mathrm{HAQ}$, but decreased to approximately $50 \%$ for absence of fatigue and did not differ according to the SR definition used. Fatigue is a symptom that patients consider to be as severe as pain, and they frequently experience fatigue as incontrollable and overwhelming [37]. Among patients who achieved remission (or low disease activity), fatigue is frequent and may be experienced as a problem that affects quality of life [38-40]. Additionally, a relationship between female sex and high levels of fatigue has been documented in a systematic review [41]. Our cohort was highly represented by females, which may explain the considerable prevalence of substantial fatigue in patients with SR, a number that was independent of the stringency of the SR definition used.

Fourth, we identified a particular temporal pattern of outcome normalization in patients who achieved SR, characterized by consecutive (rather than simultaneous) patient-centered followed by physician-centered, outcome normalization, with only mild differences between both SR definitions. Acute reactant phase normalization was detected very early during follow-up, followed by the simultaneous normalization of the majority of PRO, physician-dependent outcomes (as were swollen joint count and overall disease VAS) and SR construct. Moreover, 7 to 9 months extended between the normalization of acute reactant phases and the time point when the SR criteria are achieved. The results emphasize that the impact of SR on serologic disease activity, PRO and clinical disease activity do not necessarily occur in parallel, as previously highlighted by Altawil et al. [42]. In addition, SR according to DAS28 and the Boolean definition had a similar degree of stringency regarding their gain in most of the outcomes relevant to patients. In terms of fatigue and the physical component of the SF-36, it did behave similarly, but absence of fatigue and normalization of the physical component of the SF-36 were achieved earlier when the Boolean definition was used. The most strict definition translated into (2 specific) earlier improved patient-reported long-term outcomes. We do not have a clear explanation for the behavior of those outcomes. Nonetheless, Thiele et al. compared the functional ability of German RA patients in remission according to the DAS28, the SDAI and the Boolean definition of remission; they showed small differences between the 3 remission groups in swollen joint counts, moderate differences in acute phase reactants but large differences in PRO; patients achieving Boolean remission had a better patient global assessment as well as lower pain and fatigue scores than those in SDAI remission meanwhile for patients with DAS28 remission, disease duration, pain, fatigue and comorbid spine disease were associated with not achieving the other 2 sets of remission criteria [26].

The limitations of the study should be considered when interpreting our results. B and DAS-28 -SR's definition include a patient VAS that can be high in patients with comorbid musculoskeletal conditions given that patients may not be able to distinguish between discomfort due to comorbid conditions and due to RA. Comorbid conditions were highly prevalent in our cohort [18]. We applied DAS28-SR using DAS28-ESR. An association between the ESR level and age [43] has been described, and elderly patients ( $3.2 \%$ of our cohort had $\geq 65$ years of age) with true SR may have been misclassified as with some disease activity. Also, age (and gender) influence normative data of PROs (as SF-36 and HAQ); nonetheless, older age was underrepresented in our cohort and its impact on SR assessments is likely to be minimal. Fatigue was assessed using the fatigue short form 36 vitality subscale instead of a validated scale or an appropriated questionnaire primarily designed to assess fatigue [44]. Our cohort of patients is highly represented by females as previously described $[17,18]$ and data reported should not be generalized to RA males. Finally, additional confounding factors highly prevalent in RA patients [45] with confirmed strong associations with PRO, such as depression and anxiety, were not possible to control for given that these factors were not part of the original program in the patient's follow-up.

\section{Conclusions}

In conclusion, awareness of the patient preferences is a salient premise for priorities in health care as it has been 
demonstrated that patients and health professionals differ in their perceptions of patients' health status and need for care [46, 47]. Remission status is the most desirable outcome. For adoption by both patients and physicians, remission status should reflect symptom's resolution and ideally prevent subsequent structural damage. PRO provide unique information that cannot be collected from a physician, especially regarding symptoms, and complete the picture of patients who live with RA in the clinical context of sustained remission.

\section{Abbreviations}

ACCP: Antibodies to cyclic citrullinated proteins; ACR: American Colleague of Rheumatology; B-SR: Boolean definition of sustained remission; CRP: Creactive protein; DAS28: Disease activity score on 28 joints; DMARDs: Disease modifying anti-rheumatic drugs; EAC: Early Arthritis Clinic; ESR: Erythrocyte sedimentation rate; EULAR: European League Against Rheumatism; HAQ: Health assessment questionnaire; HAQ-N: Health assessment questionnaire- norm; HRQoL: Health-related quality of life; OMERACT: Outcomes measures in Rheumatology; O-VAS: Overall diseasevisual analogue scale; OVAS-N: Overall disease-visual analogue scale-norm; PhyVAS-N: Physician overall disease visual analogue scale normalization; PRO: Patient-reported outcomes; P-VAS: Pain visual analogue scale; PVAS$\mathrm{N}$ : Pain visual analogue scale-norm; RA: Rheumatoid arthritis; RF: Rheumatoid factor; SF-36-N: Short-Form 36v2 Survey-norm; SR: Sustained remission; SR36: Short-Form 36v2 Survey

\section{Acknowledgements}

None.

\section{Funding}

None.

\section{Availability of data and materials}

All data that support our findings are contained within the manuscript. Requests for further details on the dataset and queries related to data sharing arrangements may be submitted to the corresponding author.

\section{Authors'contributions}

ICY: Participated in the conception and design of the study and performed the statistical analysis. In charge of databases integrity of the early arthritis clinic. GG: Participated in the conception of the study and reviewed the manuscript. DRD: Participated in the conception of the study and reviewed the manuscript. VPR: Participated in the conception and design of the study; performed the statistical analysis and drafted the manuscript. In charge of the early arthritis clinic; performed patient's clinical evaluations. All authors read and approved the final manuscript.

\section{Authors' information}

All authors read and approved this manuscript.

\section{Ethics approval and consent to participate}

The study was approved by the Institutional Review Board of the Instituto Nacional de Ciencias Médicas y Nutrición Salvador Zubirán with the reference number IRE-274-10/11-1. All necessary consent approval was obtained from any patients involved in the study.

\section{Consent for publication}

Not applicable.

\section{Competing interests}

The authors declare that they have no financial interests, which could create a potential conflict of interest with regard to the work.

\section{Publisher's Note}

Springer Nature remains neutral with regard to jurisdictional claims in published maps and institutional affiliations.

\section{Author details}

'Department of Immunology and Rheumatology, Instituto Nacional de Ciencias Médicas y Nutrición Salvador Zubirán, Vasco de Quiroga 15, Colonia Belisario Domínguez, Sección XVI, 14080 México City, CP, Mexico.

${ }^{2}$ Department of Internal Medicine, Instituto Nacional de Ciencias Médicas y Nutrición Salvador Zubirán, Vasco de Quiroga 15, Colonia Belisario Domínguez, Sección XVI, 14080 México City, CP, Mexico.

Received: 23 March 2017 Accepted: 11 August 2017

Published online: 02 September 2017

\section{References}

1. Aletaha D, Funovits J, Breedveld FC, Sharp J, Segurado O, Smolen J. Rheumatoid arthritis joint progression in sustained remission is determined by disease activity levels preceding the period of radiographic assessment. Arthritis Rheum. 2009;60:1242-9.

2. van der Heijde D. Remission by imaging in rheumatoid arthritis: should this be the ultimate goal? Ann Rheum Dis 2012;71(Supll):i89-i92. Doi:10.1136/ annrheumdis-2011-200797.

3. Makinen $\mathrm{H}$, Kautiainen $\mathrm{H}$, Hannonen P, Sokka T. Is DAS28 an appropriated tool to assess remission in rheumatoid arthritis? Ann Rheum Dis. 2005;64:1410-3.

4. Felson DT, Smolen JS, Wells G, Zhang B, van Tuyl LH, Funovits J, et al. American College of Rheumatology/European league against rheumatism provisional definition of remission in rheumatoid arthritis for clinical trials. Ann Rheum Dis. 2011;70:404-13.

5. Pinals RS, Masi AT, Larsen RA. Preliminary criteria for clinical remission in rheumatoid arthritis. Arthritis Rheum. 1981;24:1308-15.

6. Felson D. Defining remission in rheumatoid arthritis. Ann Rheum Dis 2012; 71(0 2): i86-i88. Doi:10.1136/annrheumdis-2011-200618.

7. West $\mathrm{E}$, Jonsson SW. Health-related quality of life in rheumatoid arthritis in northern Sweden; a comparison between patients with early RA, patients with medium-term disease and controls, using SF-36. Clin Rheumatol. 2005;24:117-22.

8. Linde I, Sorensen J, Ostergaad M, Horslev-Petersen K, Hetland ML. Does clinical remission lead to normalization of EQ-5D in patients with rheumatoid arthritis and is selection of criteria important? J Rheumatol 2010;37:285-290.

9. Kahn KL, Mclean CH, Wong AL, Rubenstein LZ, Liu H, Fitzpatrick DM, et al. Assessment of American College of Rheumatology Quality Criteria for rheumatoid arthritis in a pre-quality criteria patient cohort. Arthritis Rheum. 2007:57:707-15.

10. Hewlett S, Carr M, Ryan S, Kirwan J, Richards P, Carr A, et al. Outcomes generated by patients with rheumatoid arthritis: how important are they? Musculoskeletal Care. 2005;3:131-42.

11. Sanderson T, Kirwan J. Patient-reported outcomes of arthritis: time to focus on personal life impact measures? Editorial Arthritis Rheum. 2009;61:1-3.

12. Hewlett S. Patients and clinicians have different perspectives on outcomes in arthritis. J Rheumatol. 2003;30:877-9.

13. Charlson M, Szatrowsky TP, Peterson J, Gold J. Validation of a combined comorbidity index. J Clin Epidemiol. 1994:47(11):1245-51.

14. Ramey DR, Raynauld JP, Fries JF. The health assessment questionnaire 1992: status and review. Arthritis Care Res. 1992:5:119-29.

15. Ware JE Jr. SF-36 health survey update. Spine. 2000;25:3130-9.

16. Maruish ME (Ed). User's manual for the SF-36v2 health survey (3rd edition) 2011. Lincoln Rl: Qualitimetric Incorporated.

17. Contreras-Yánez I, Pascual-Ramos V. Window of opportunity to achieve major outcomes in early rheumatoid arthritis patients: how persistence with therapy matters. Arthritis Res Ther 2015;17:177. DOI 10.1186/s13075-015-0697-z.

18. Contreras-Yánez I, Rull-Gabayet M, Pascual-Ramos V. Early disease activity suppression and younger age predict excellent outcome on recent-onset rheumatoid arthritis patients with conventional disease modifying antirheumatic drugs. Clin Exp Rheumatol. 2012;30:402-8.

19. Prevoo ML, van't Hof MA, Kuper $H H$, van Leeuwen $M A$, van de Putte $L B$, van Riel PL: Modified disease activity scores that include twenty-eight-joint counts. Development and validation in a prospective longitudinal study of patients with rheumatoid arthritis. Arthritis Rheum 1995:38:44-48.

20. Krishnan E, Sokka T, Häkkinen A, Hubert H, Hannonen P. Normative values for the health assessment questionnaire disability index: benchmarking disability in the general population. Arthritis Rheum. 2004 Mar;50(3):953-60.

21. Durán-Arenas L, Gallegos-Carrillo K, Salinas-Escudero G, Martínez-Salgado H. Hacia una base normativa mexicana en la medición de calidad de vida relacionada con la salud, mediante el Formato Corto 36. Salud Publica Mex. 2004;46:306-15 
22. Fransen J, van Riel PLCM. DAS remission cut points. Clin Exp Rheumatol. 2006;24:S29-32.

23. Bird $P$, Nicholls $D$, Barrett $R$, Jager JD, Griffiths $H$, Roberts $L$, et al. Longitudinal study of clinical prognostic factors in patients with early rheumatoid arthritis: the PREDICT study. Int J Rheum Dis. 2017;20(4):460-8.

24. Radner H, Smolen JS, Aletaha D. Remission in rheumatoid arthritis: benefit over low disease activity in patient-reported outcomes and costs. Arthritis Res Ther. 2014;16:R56.

25. Js S, Aletaha D, Bijlsma JW, Breedveld FC, Boumpas D, Burmester G, et al. Treating rheumatoid arthritis to target: recommendations of an international task force. Ann Rheum Dis. 2010;69:631-7.

26. Thiele K, Huscher D, Bischoff SL, Späthling-Mestekemper S, Backhaus M, Aringer M, et al. Performance of the 2011 ACR/EULAR preliminary remission criteria compared with DAS28 remission in unselected patients with rheumatoid arthritis. Ann Rheum Dis. 2013;72:1194-9.

27. Kirkham JJ, Boers M, Tugwell P, Clarke M, Williamson PR. Outcome measures in rheumatoid arthritis randomized trials over the last 50 years. Trials. 2013;14:324.

28. Gossec L, Dougados M, Dixon W. Patient-reported outcomes as end points in clinical trials in rheumatoid arthritis. RMD Open 2015;1:e000019.doi:10. 1136/rmdopen-2014-000019.

29. Bykerk V, Massarotti EM. The new ACR/EULAR remission criteria: a rationale for developing new criteria for remission. Rheumatology 2015;51:vi16-vi20. Doi:10.1093/rheumatology/kes281.

30. Kuriya B, Sun Y, Boire G, Haraoui B, Hitchon C, Pope JE, et al. Canadian early arthritis cohort. Remission in early rheumatoid arthritis- a comparison of new ACR/EULAR remission criteria to established criteria. J Rheumatol. 2012; 39:1155-8.

31. Sakellariou G, Scire CA, Verstappen SM, Montecucco C, Carporali R. In patients with early RA the new ACR/EULAR definition of remission identifies patients with persistent absence of functional disability and suppression of ultrasonographic synovitis. Ann Rheum Dis. 2013;72:245-9.

32. Aletaha D, Ward MM, Machold KP, Nell VPK, Stamm T, Smolen JS. Remission and active disease in rheumatoid arthritis: defining criteria for disease activity states. Arthritis Rheum. 2005;52:2625-36.

33. Aletaha D, Smolen J, Ward MM. Measuring function in rheumatoid arthritis: identifying reversible and irreversible components. Arthritis Rheum. 2005;54:2784-92.

34. Hillsdon MM, Brunne EJ, Guralnik JM, Marmot MG. Prospective study of physical activity and physical function and early old age. Am J Prev Med. 2005;28:245-50.

35. Jakobsson U, Hallberg IT. Pain and quality of life among older people with rheumatoid arthritis and/or osteoarthritis: a literature review. J Clin Nurs. 2002;11:430-43.

36. Radner H, Alasti F, Smolen JS, Aletaha D. Physical function continues to improve when clinical remission is sustained in rheumatoid arthritis patients. Arthritis Res Ther. 2015;17:203.

37. Hewlett S, Chalder T, Choy E, Cramp F, Davis B, Dures E, et al. Fatigue in rheumatoid arthritis: time for a conceptual model. Rheumatology (Oxford). 2011;50:1004-6.

38. Curtis JR, Shan Y, Harrold L, Zhang J, Greenberg JD, Reed GW. Patient perspectives on achieving treat to target goals: a critical examination of patient-reported outcomes. Arthritis Care Res (Hoboken). 2013;65:1707-12.

39. Van Hoogmoed D, Fransen J, Repping-Wuts H, Spee L, Bleijenberg G, van Riel PL. The effects of anti-TNF versus DMARDs on fatigue in rheumatoid arthritis patients. Scan J Rheumatol. 2013:42:15-9.

40. Olsen $C L$, Lie E, Kvien TK, Zangi HA. Predictors of fatigue in rheumatoid arthritis patients in remission or in low disease activity. Arthritis Car Res. 2016;68:1043-8.

41. Nikolaus S, Bode C, Taal E, van de Laar MA. Fatigue and factors related to fatigue in rheumatoid arthritis: a systematic review. Arthritis Car Res (Hoboken). 2013;65:1128-46.

42. Altawil R, Saevarsdottir S, Wedren S, Alfredsson L, Klareskog L, Lampa J. Remaining pain in early rheumatoid arthritis patients treated with methotrexate. Arthritis Car Res. 2016;68:1061-8.

43. Lane SK, Gravel JW Jr. Clinical utility of common serum rheumatologic tests. Am Fam Physician. 2002 Mar 15;65:1073-80.

44. Hewlett S, Dures E, Almeida C. Measures of Fatigue. Bristol Rheumatoid Arthritis Fatigue Multi-Dimensional Questionnaire (BRAF MDQ), Bristol Rheumatoid Arthritis Fatique Numerical Rating Scales (BRAF NRS) for Severity, Effect, and Coping, Chalder Fatigue Questionnaire (CFQ), Checklist
Individual Strength, (CIS2OR and CIS8R), Fatigue Severity Scale (FSS), Functional Assessment Chronic Illness, Therapy (Fatigue) (FACIT-F), MultiDimensional Assessment of Fatigue (MAF), Multi-Dimensional Fatigue Inventory (MFI), Pediatric Quality Of Life (PedsQL) Multi-Dimensional Fatigue Scale, Profile of Fatigue (ProF), Short Form 36 Vitality Subscale (SF-36 VT), and Visual Analog Scales (VAS). Arthritis Car Res 2011;63:S263.86.

45. Covic T, Cumming SR, Pallant JF, Manolios N, Emery P, Conaghan PG, et al. Depression and anxiety in patients with rheumatoid arthritis: prevalence rates based on a comparison of the depression, anxiety and stress scale (DASS) and the hospital anxiety and depression scale (HADS). BMC Psychiatry. 2012;12:6

46. Berkanovic E, Hurwicz ML, Lachewnbruck CA. Concordant and discrepant views of patient's physical functioning. Arthritis Car Res. 1995;8:94-101.

47. Molzhan AE, Northcott HC. The social bases of discrepancies in health/ illness perceptions. J Adv Nurs. 1989;14:132-40.

\section{Submit your next manuscript to BioMed Central and we will help you at every step:}

- We accept pre-submission inquiries

- Our selector tool helps you to find the most relevant journal

- We provide round the clock customer support

- Convenient online submission

- Thorough peer review

- Inclusion in PubMed and all major indexing services

- Maximum visibility for your research

Submit your manuscript at www.biomedcentral.com/submit
Biomed Central 